\title{
Fulminant gastrointestinal hemorrhage due to Strongyloides stercoralis hyperinfection in an AIDS patient
}

\author{
Valdes Roberto Bollela ${ }^{[1]}$, Cinara Feliciano ${ }^{[1]}$, André Costa Teixeira ${ }^{[2]}$, \\ Ana Carolina Ribeiro Junqueira ${ }^{[1]}$ and Marcos Antonio Rossi ${ }^{[2]}$
}

[1]. Divisão de Moléstias Infecciosas e Tropicais, Departamento de Clínica Médica, Faculdade de Medicina de Ribeirão Preto, Universidade de São Paulo, Ribeirão Preto, SP. [2]. Departamento de Patologia e Medicina Legal, Faculdade de Medicina de Ribeirão Preto, Universidade de São Paulo, Ribeirão Preto, SP.

\begin{abstract}
Strongyloides stercoralis is an endemic nematode to tropical and subtropical regions of the globe. The parasite is capable of autoinfection, which is limited by an intact immune response. In immunocompromised hosts, hyperinfection and dissemination can occur and have a high index of mortality. A hyperinfection syndrome with dissemination is frequently associated with corticosteroid administration and other conditions (malignancies and organ transplantation). Interestingly, although strongyloidiasis is common among AIDS patients in endemic areas, the hyperinfection syndrome is rarely noted. We report here on a rare manifestation of fulminant gastrointestinal hemorrhage due to hyperinfection of strongyloidiasis in a female drug-abusing, alcoholic HIV/AIDS patient.
\end{abstract}

Keywords: Strongyloides stercoralis.Gastrointestinal hemorrhage. AIDS.

\section{INTRODUCTION}

Strongyloides stercoralis is a nematode endemic to humid, tropical regions including Africa, Southeast Asia, and Latin America. This worm is unique among helminths that commonly cause infections because it can replicate within the human host $^{1}$. Patients are usually asymptomatic and able to control the proliferation of the larvae, though not eradicate them. The parasite is capable of autoinfection, which is limited by an intact immune response. A low level of autoinfection may allow the organism to persist for decades and cause clinical manifestations long after the initial infection ${ }^{2}$.

In immunocompromised hosts, hyperinfection and dissemination can occur, which has a high mortality. Dissemination of $S$. stercoralis is potentially lethal as it is often associated with bacterial sepsis. Although patients may survive if diagnosed at an early stage, the hyperinfection syndrome has a mortality rate ranging from $15 \%$ to $87 \%$.

The diagnosis of disseminated strongyloidiasis is often delayed because of nonspecific presenting symptoms. Early diagnosis relies on a high index of clinical suspicion, especially in immunocompromised hosts ${ }^{4}$. The hyperinfection syndrome with dissemination is frequently associated with

Address to: Dr. Valdes Roberto Bollela. Divisão de Moléstias Infecciosas e Tropicais/ HC/FMRP. Av. Bandeirantes 3900, Monte Alegre, 14049-900 Ribeirão Preto, SP, Brasil.

Phone: 5516 3602-2468

e-mail: vbollela@gmail.com

Received in 27/02/2012

Accepted in 20/06/2012 corticosteroid administration. Other conditions associated with the hyperinfection syndrome include malignancies and organ transplantation ${ }^{5}$. Interestingly, although strongyloidiasis is common among AIDS patients in endemic areas, the hyperinfection syndrome is rarely noted. Among AIDS patients, strongyloidiasis is more commonly associated with chronic diarrhoea ${ }^{4}$. We report here on a rare manifestation of fulminant gastrointestinal hemorrhage due to hyperinfection of strongyloidiasis in a female drug-abusing, alcoholic human immunodeficiency virus/acquired immunodeficiency syndrome (HIV/AIDS) patient.

\section{CASE REPORT}

A 24-years-old woman, diagnosed with the human immunodeficiency virus for 10 years, with a poor adherence to antiretroviral therapy and followed in an HIV/AIDS specialized ambulatory, was referred to the hospital complaining about an intense and diffuse abdominal pain associated with diarrhoea for 20 days, nausea and vomiting. At that moment she presented with no fever, hematemesis or black stool. She had a history of poor adherence to antiretroviral therapy, and she had missed her follow-up 6 months ago, when her cluster of differentiation 4 (CD4) count was $313 / \mathrm{mm}^{3}$ and the viral load was 153 copies/ $\mathrm{ml}$. She also had a record of schizophrenia, professional sex work, alcoholism and drug addiction (cocaine and cannabis). The patient was admitted in the Infectious Diseases ward, and her physical exam showed dehydration, emaciation and jaundice $(+/ 4+)$, and a temperature of $37^{\circ} \mathrm{C}$. She had intense oral candidiasis, tachycardia and hypotension. There were no abnormalities in the chest exam, but she complained of pain 
during the abdominal exam, especially on the upper right side. There was no sign of peritoneum inflammation in the physical exam. The Glasgow coma scale was 15 .

\section{Complementary tests}

Thoracic and abdominal radiography were normal. Hemoglobin: 9.0g/dl; leucocytes: 19,200/ $\mu$ l (3\% lymphocytes); platelets: $302,000 / \mu \mathrm{l}$; total bilirubin: $2.67 \mathrm{mg} / \mathrm{dl}$ (direct: $2.19 \mathrm{mg} / \mathrm{dl}$ ); creatinine: $0.4 \mathrm{mg} / \mathrm{dl}$; serum sodium: $120 \mathrm{mmol} / 1$; potassium: $2.6 \mathrm{mmol} / 1$; amylase: $12 \mathrm{U} / \mathrm{L}$; aspartate aminotransferase (AST): 71U/L; International normalized ratio (INR): 1.69 .

Few hours after admission, the patient presented with abundant hematemesis, abdominal distension and shock. Fluids and blood transfusion were started. Nevertheless, patient failed to recover from shock and died. The blood culture at admission isolated Klebsiella pneumoniae. The necropsy showed many stomach and duodenum ulcers on the macroscopic exam. The microscopic exam revealed a countless number of Strongyloides at different stages of maturation associated with an intense inflammatory process and a diffuse ulcerative process. The parasite was not found in other organs, such as lungs and liver (Figure 1).

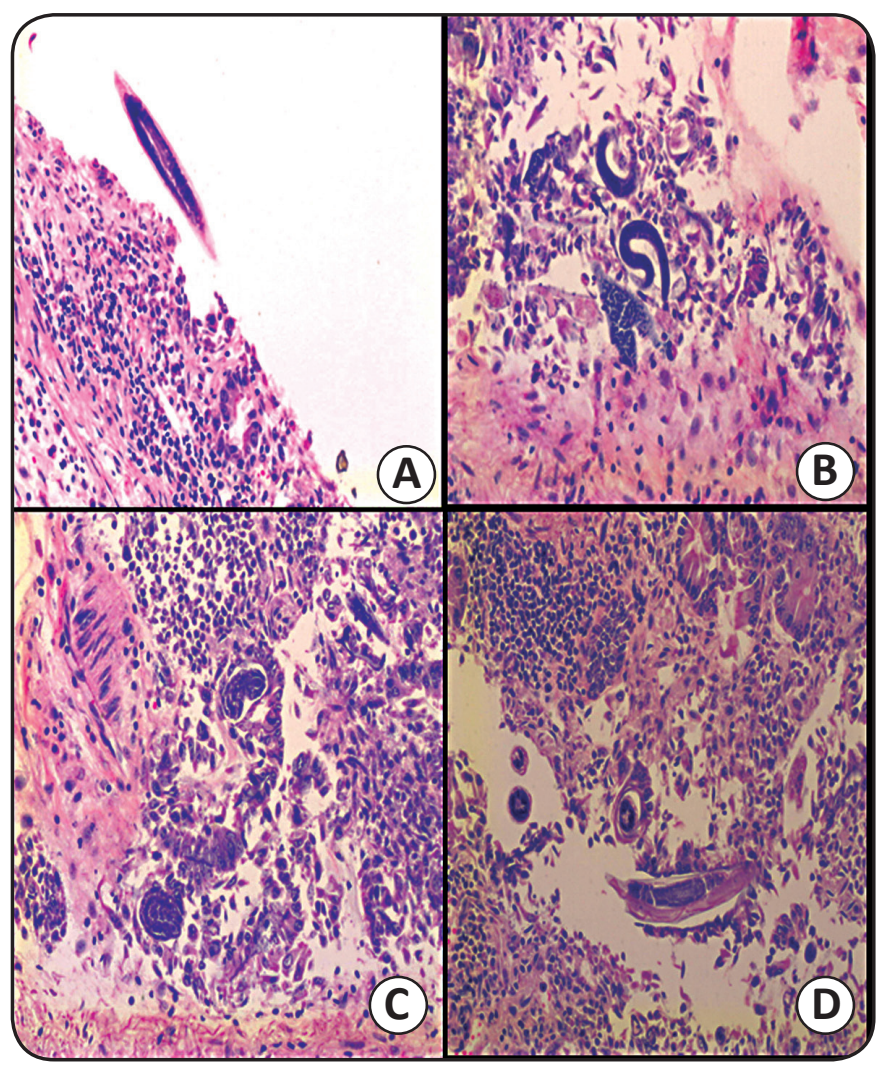

FIGURE 1 - Strongyloides worms in the gastroduodenal transition presented in different stages of maturation (A,B,C and D), associated with an intense inflammatory reaction and ulcerative mucosa.

\section{DIsCusSION}

Strongyloides stercoralis can alternate between parasitic and free-living life cycles. In the free-living cycle, the rhabditiform larvae are shed in the stool on the soil, where they grow into adult worms that produce eggs and hatch to larvae. These larvae can either grow to adult worms or penetrate the human skin to start the parasitic life cycle. In humans, the larvae are transported in the blood stream to the lungs. Through the alveoli and bronchi the larvae then reach the pharynx, where they are swallowed into the stomach and the small intestine. The larvae finally grow into adult female worms in the small intestine. By parthenogenesis, the female worms produce the eggs and larvae that cause autoinfection ${ }^{6}$.

The clinical spectrum of strongyloidiasis includes acute infection with Loeffler's syndrome, chronic intestinal infection, asymptomatic autoinfection, symptomatic autoinfection, and hyperinfection syndrome with dissemination. Immunocompetent patients usually develop an asymptomatic, or mildly symptomatic chronic infection. Chronic symptomatic infections can present with abdominal, respiratory and/or dermatological complaints (fleeting serpiginous, urticarial rash) ${ }^{4}$.

Strongyloides stercoralis infection in human hosts can be lifelong, and it can present as a hyperinfection syndrome ${ }^{7}$ in immunocompromised patients. Clinically, the hyperinfection syndrome presents with diarrhoea, gastrointestinal hemorrhage, bacteremia, pneumonia and/or meningitis. Mortality is up to $80 \%$. The most prominent risk factors for the development of the hyperinfection syndrome are glucocorticoid therapy, malnutrition and alcoholism ${ }^{3}$. Another notable clinical feature of hyperinfection is that $30-45 \%$ of cases are exacerbated by gram-negative sepsis, caused by the colonic flora invading the gut secondary to the penetration of the colonic mucosa by the Strongyloides larvae ${ }^{2}$.

The Centers for Disease Control included extra-intestinal strongyloidiasis in the original classification of opportunistic infections associated with AIDS, but this was later deleted, because only few such combinations have been recorded ${ }^{8}$. The relative infrequency of such case reports despite the vast number of people who must be co-infected is striking. There are fewer than 30 cases in the literature of hyperinfection occurring in patients infected by the HIV. A number of these patients had previously received steroids, either as adjunctive treatment for Pneumocystis jiroveci pneumonia or as part of a chemotherapeutic regimen for non-Hodgkin's lymphoma9.

In this present case, we report a Strongyloides hyperinfection in a HIV patient who was not receiving any other immunosuppressive therapy and presented two manifestations of this syndrome: an invasive and ulcerative bleeding disease in the upper gastrointestinal tract, and a Gram-negative sepsis, probably due to translocation of these bacteria in the small intestine, without opportunity for investigation and treatment.

The diagnosis of $S$. stercoralis infestation can be done by evidence of larvae and/or adult worms in the small intestinal biopsy. Duodenal aspirate examination is also a sensitive method for the diagnosis of strongyloidiasis. A definite diagnosis is made by finding one or more stages of the parasite in feces, intestinal aspirate, peritoneal fluid, peripheral blood or in tissue sections. Unfortunately, fecal larval output does not correlate with adult worm load, so it may underestimate the severity of the infection. It is also worth mentioning that the eosinophilic count is not a reliable indicator of parasitic infection. There are new tests for 
diagnosing strongyloidiasis: a serological test like the enzymelinked immunosorbent assay (ELISA), for instance, is highly sensitive (reaching up to $90 \%$ ), though not always reliable. It does not discriminate recent from old infections and produces cross-reaction with other helminth infections ${ }^{6}$. In addition, there have been reports on the use of molecular methods for the amplification of Strongyloides DNA for diagnostic purposes ${ }^{10}$.

Ivermectin or thiabendazole can be chosen to treat the S. stercoralis hyperinfection syndrome. Ivermectin is much better tolerated and has been used successfully in cases that did not respond adequately to thiabendazole. Two doses of ivermectin are given 1-14 days apart, which has a cure rate of $94-100 \%$. Albendazole can be used as an alternative if nothing else is available, as it has a lower efficacy $(38-45 \%)^{4}$.

The combination of Strongyloides hyperinfection and AIDS can be diagnosed by clinicians aware of this possibility, and fatal consequences of this uncommon condition can be avoided.

\section{REFERENCES}

1. Grove DI. Strongyloidiasis: a conundrum for gastroenterologists. Gut 1994; 35:437-440.
2. Lam CS, Tong MKH, Chan KM, Siu YP. Disseminated strongyloidiasis: a retrospective study of clinical course and outcome. Eur J Clin Microbiol Infect Dis 2006; $25: 14-18$

3. Marcos LA, Terashima A, Dupont HL, Gotuzzo E. Strongyloides hyperinfection syndrome: an emerging global infectious disease. Trans R Soc Trop Med Hyg 2008; 102:314-318.

4. Montes M, Sawhrey C, Barros N. Strongyloides stercoralis: there but not seen. Curr Op Infect Dis 2010; 23:500-504

5. Machado ER, Teixeira EM, Goncalves-Pires MDRF, Loureiro ZM, Araújo RA, Costa-Cruz JM. Parasitological and immunological diagnosis of Strongyloides stercoralis in patients with gastrointestinal cancer. Scand J Infect Dis 2008; 40:154-158.

6. Agrawal V, Agarwal T, Ghoshal UC. Intestinal strongyloidiasis: a diagnosis frequently missed in the tropics. Trans R Soc Trop Med Hyg 2009; 103:242-246.

7. Veloso MGP, Porto AS, Moraes M. Strongyloides stercoralis hyperinfection: autopsy case report. Rev Soc Bras Med Trop 2008; 41:413-415.

8. Harcourt-Webster JN, Scaravilli F, Darwish JN, Harcourt-Webster F, Scaravilli A, Darwish H. Strongyloides stercoralis hyperinfection in an HIV positive patient. J Clin Pathol 1991; 44:346-348.

9. Keiser PB, Nutman TB. Strongyloides stercoralis in the immunocompromised population. Clin Microbiol Rev 2004; 17:208.

10. Kia EB, Rahimi HR, Mirhendi H, Nilforoushan MR, Talebi A, Zahabiun F, et al. A case of fatal strongyloidiasis in a patient with chronic lymphocytic leukemia and molecular characterization of the isolate. Korean J Parasitol 2008; 46:261-263. 